\title{
mTORC is a potential therapeutic target in APS
}

A new study published in the New England Journal of Medicine suggests that the mammalian target of rapamycin complex (mTORC) affects vasculopathy in patients with antiphospholipid syndrome (APS).

Circulating autoantibodies in patients with APS cause fibrosis, thrombotic events and vascular cellular infiltrates which often lead to life-threatening complications such as myocardial infarction or renal failure. "Vasculopathy associated with APS was already fairly well described in many organs, but the molecular pathways involved were unknown", says author Dr Fabiola Terzi (INSERM U1151, Université Paris Descartes, France).

In a set of experiments focused on the identification of these molecular pathways, Terzi and colleagues compared samples from a cohort of patients with APS, SLE or both diseases, together with a previously described cohort of patients who received a kidney transplant and had antiphospholipid antibodies or not.

The authors showed that vessels from patients with APS nephropathy had increased mTORC activation. Importantly, when compared with control IgG antibodies, polyclonal antiphospholipid antibodies induced significantly higher phosphorylation of mTORC pathway members S6RP and AKT in a human microvascular endothelial cell line, a process that was dependent on the phosphatidylinositol 3-kinase pathway.

Further evidence of the importance of the mTORC pathway in APS vasculopathy was provided by analysis of patients with kidney transplants: among those with antiphospholipid antibodies, 7 out of $10(70 \%)$ patients who received the mTOR inhibitor sirolimus (also known as rapamycin) as immunosuppressive therapy had well preserved kidney function 144 months after transplantation, whereas only 3 out of 27 (11\%) patients who were not treated with sirolimus had functional kidneys at that time.

The processes by which antiphospholipid antibodies activate mTORC is still mostly unknown, but the authors intend to

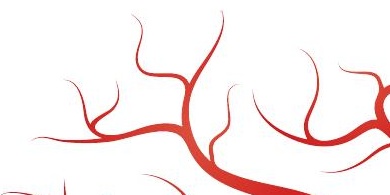
"identify the receptors of antiphospholipid antibodies that activate the AKT/mTORC pathway in endothelial cells". Terzi adds that these findings "uncovered a new therapeutic strategy for the treatment of the most severe forms of APS" and "open a new field of investigation on how antiphospholipid antibodies may damage vessels leading to organ failure".

\section{João H. Duarte}

Original article Canaud, G. et al. Inhibition of the mTORC pathway in the antiphospholipid syndrome. N. Engl. J. Med. doi:10.1056/nejmoa1312890 